\title{
Precision Method for Evaluating Primary Aberrations of Lenses With a Twyman Interferometer
}

\author{
James B. Saunders
}

\begin{abstract}
(May 21, 1965)
A simplified procedure for separate evaluation of the three primary aberrations of lenses with the Twyman lens testing interferometer is described. Each of the aberration coefficients is found to be a function of observations at only four points on the lens. Equations are given for the optimum choice of reference points that give best results. These equations are applied to data previously reported in the literature. The results indicate that this procedure is sufficiently precise to reveal high order aberrations in a lens that was assumed to be practically free from such aberrations.
\end{abstract}

Key Words: Interferometer, lens testing, Twyman interferometer.

\section{Introduction}

The formula for optical path difference in the Twyman lens testing interferometer, used by Professor Kingslake, contains seven parameters. It would seem that data from seven reference points would be necessary for evaluating any one of the parameters. By judiciously choosing the location of the reference points each of the primary monochromatic aberrations may be evaluated from data taken at only four points. It will be shown that the results obtained from four points, with a given lens, are of the same order of accuracy as those previously obtained, by least squares, from 44 points. This represents a very large reduction in computations.

Each of the primary monochromatic aberrations is separately evaluated. The results indicate that the sensitivity of the Twyman interferometer is sufficient, not only to measure the aberration coefficients accurately, but also to measure changes in these aberrations with changes in the aperture of the lens.

The discovery that four properly chosen points are adequate for evaluation of each aberration coefficient with the Twyman interferometer was the result of an anologous discovery that two points are adequate for the same measurements with the Kösters prism interferometer.

\section{Fundamental Equations}

The terminology previously used by Kingslake ${ }^{1}$ will be used in this paper. The data which he obtained with a simple lens ${ }^{2}$ will be used for demonstration of the procedures described here. This lens was as. sumed to be practically free from higher order aberrations over the aperture used for obtaining results.

${ }^{1} \mathrm{R}$. Kingslake, The interferometer patterns due to the primary aberrations, Trans. Opt. Soc. 27, 94 (1925-6).

${ }^{2}$ R. Kingslake, The analysis of an interferogram, Trans. Opt. Soc. 28, 1 (1926-7)
The formula for optical path difference is

$$
\begin{aligned}
A\left(x^{2}+y^{2}\right)^{2}+B y\left(x^{2}+\right. & \left.y^{2}\right)+C\left(x^{2}+3 y^{2}\right) \\
& +D\left(x^{2}+y^{2}\right)+E y+F x+G=p
\end{aligned}
$$

where $A, B$, and $C$ represent, respectively, coefficients of spherical aberration, coma, and astigmatism. These coefficients are related to the aberration constants, $a_{1}, a_{2}$, and $a_{3}$, by the equations,

$$
\left.\begin{array}{c}
4 A=a_{1}=\frac{\begin{array}{c}
\text { Longitudinal (primary) } \\
\text { spherical aberration }
\end{array}}{s^{2} f^{2}} \\
f B / h=a_{2}=\frac{\text { coma }}{s^{2} h} \\
2 f^{2} C / h^{2}=a_{3}=\frac{\text { distance between focal lines }}{2 h^{2}}
\end{array}\right\}
$$

where $s$ is half the aperture of the lens, $h$ is the image distance from the optic axis and $f$ is the distance from the image to the back surface of the lens. The $x y$ rectangular coordinate system, used in eq (1), is chosen so that the $x y$-plane is in the principal plane of the lens - with the $y$-axis in the meridional plane. The origin is on the optic axis of the lens. The coefficients, $D, E, F$, and $G$ relate to adjustments of the interferometer. The order of interference is $G$, at the origin (on the axis of the lens), and is $p$ at any point $(x, y)$.

Figure 1 shows a photograph of the interferogram used by Kingslake in footnote 2. This interferogram contains the effects of any and all aberrations that are associated with this lens when it is used in the manner described. If this interferogram should contain higher order aberrations, eq (1) could not represent the fringes. The parameters, $A, B$, and $C$ would be found to vary with the aperture of the lens; whereas, 


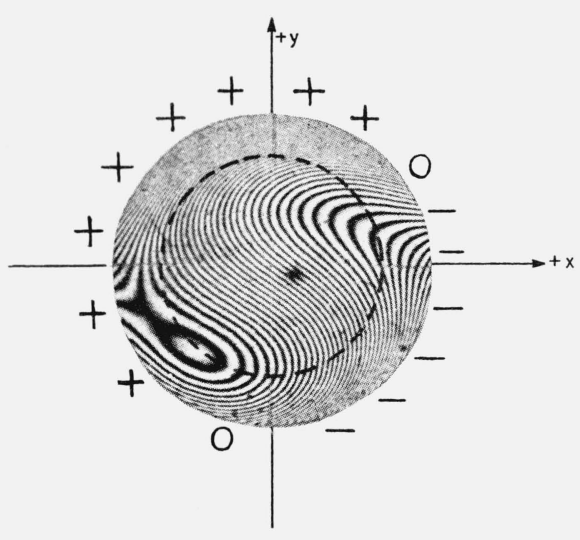

FIGURE 1. Interference fringes of a simple ophthalmic lens as seen in a Twyman lens testing interferometer.

The inscribed circle represents the "useful aperture of the lens." This area was previously assumed to be practically free from higher order aberrations. The $(+)$ and $(-)$ signs, about the margin, represent regions of positive and negative orders of interference.

if the lens is free from higher order aberrations these parameters would be independent of lens aperture and, therefore, also independent of the location of the chosen reference points.

The family of curves in figure 2 represents the interferogram of a lens that is absolutely free from higher order aberrations. These curves (fringes) are computed from eq (1) and represent the area inside the circle in figure 1. The values for the parameters are those obtained by Kingslake (see footnote 2). Each curve corresponds to an integral order of interference (integral value for $p$ ).

\section{Choice of Reference Points}

Data from a family of at least four reference points are required to obtain a unique value for each of the aberration coefficients, $A, B$, and $C$ in eq (1). The relative distribution of these points (fig. 3) can be so chosen that when the observed data (values for $x, y$, and $p$ ) at four points are substituted for their counterparts in eq (1), the resultant four equations will permit the elimination of all parameters except the one desired. There are many different arrangements of these four reference points that will permit solutions of the aberration parameters. Preliminary study showed that, in each case, one such arrangement of points could be found that yields optimum accuracy. Therefore, only one arrangement of points will be given here for evaluating each of the three aberration parameters. These arrangements are those which give optimum accuracy.

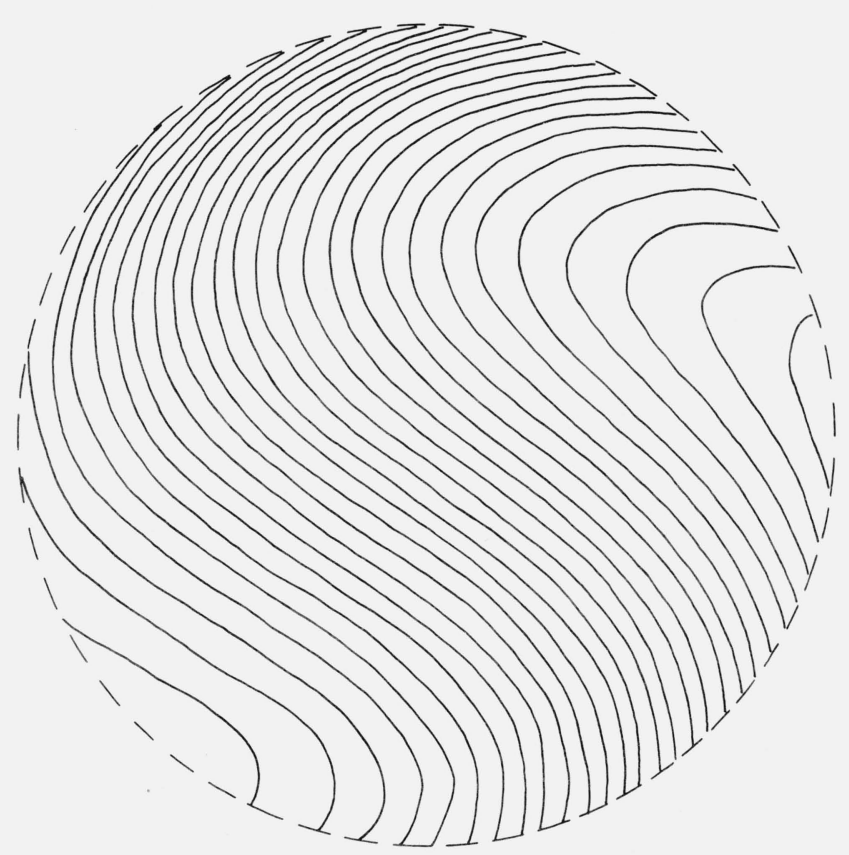

FIGURE 2. Graphical representation of computed fringes inside the circle of figure 1.

These curves were computed from the optical path difference formula (eq (1)) for a lens These curves were computed from the
that contains only primary aberrations. 


\section{Astigmatism}

If we draw a circle of radius $r$, with its center at the origin, in figure 3 , it will intersect the coordinate axes at $(r, 0)(0, r),(-r, 0)$, and $(0,-r)$. If we choose these four points in the interferogram of figure 1 , we may evaluate the parameter $C$ from data observed at these points.

The observed data are the orders of interference, $p$, and the coordinates, $x$ and $y$, at the four reference points. The data, labeled $\left(x_{1}, y_{1}, p_{1}\right),\left(x_{2}, y_{2}, p_{2}\right)$, $\left(x_{3}, y_{3}, p_{3}\right)$, and $\left(x_{4}, y_{4}, p_{4}\right)$, may be considered as the coordinates in a three dimension rectangular coordinate system. We get optimum accuracy when the following relationships exist between the $x$ and $y$ coordinates:

$$
\begin{aligned}
& x_{1}=y_{2}=-x_{3}=-y_{4}=r, \\
& y_{1}=x_{2}=y_{3}=x_{4}=0 .
\end{aligned}
$$

On substituting these values in eq (1), successively for the four points, we obtain the following four equations:

$$
\left.\begin{array}{rrr}
r^{4} A+r^{2} C+r^{2} D+r F+G=p_{1} \\
r^{4} A+r^{3} B+3 r^{2} C+r^{2} D+r E+G=p_{2} \\
r^{4} A+r^{2} C+r^{2} D-r F+G=p_{3} \\
r^{4} A-r^{3} B+3 r^{2} C+r^{2} D-r E+G=p_{4}
\end{array}\right\} .
$$

If we add the first equation in 3 to the third and the second to the fourth, we obtain,

$$
\left.\begin{array}{l}
2 A r^{4}+2 C r^{2}+2 D r^{2}+2 G=p_{1}+p_{3} \\
2 A r^{4}+6 C r^{2}+2 D r^{2}+2 G=p_{2}+p_{4}
\end{array}\right\} .
$$

By subtracting the first member equation of 4 from the second member we obtain

$$
4 C r^{2}=p_{2}+p_{4}-p_{1}-p_{3} .
$$

Values for $x$ and $y$, along the two axes, are given by Kingslake for integral values of $p$. These values are represented graphically in figure 4 . The values for the $p$ 's in table 1 are interpolated from figure 4 . When the $p$ 's, that correspond to any chosen value for $r$, are substituted in eq (5) we obtain corresponding values for $C$. These values are given in column 6 , table 1 . The probable error in $C$ is given in column 7 as $\Delta C$, and is based on the assumption that the probable error in a single observation is the same as that obtained by Kingslake. The probable error, $\Delta P$, of a single observation was found to be $0.013 \lambda$. Values for $C$ are plotted in figure 5 as ordinates for corresponding values of $r$ as abscissas. The magnitude of the probable error, $\Delta C$, is represented by the length of arrows attached to the corresponding points. Obviously, the most precise value for $C$ is obtained by using large values for $r$.

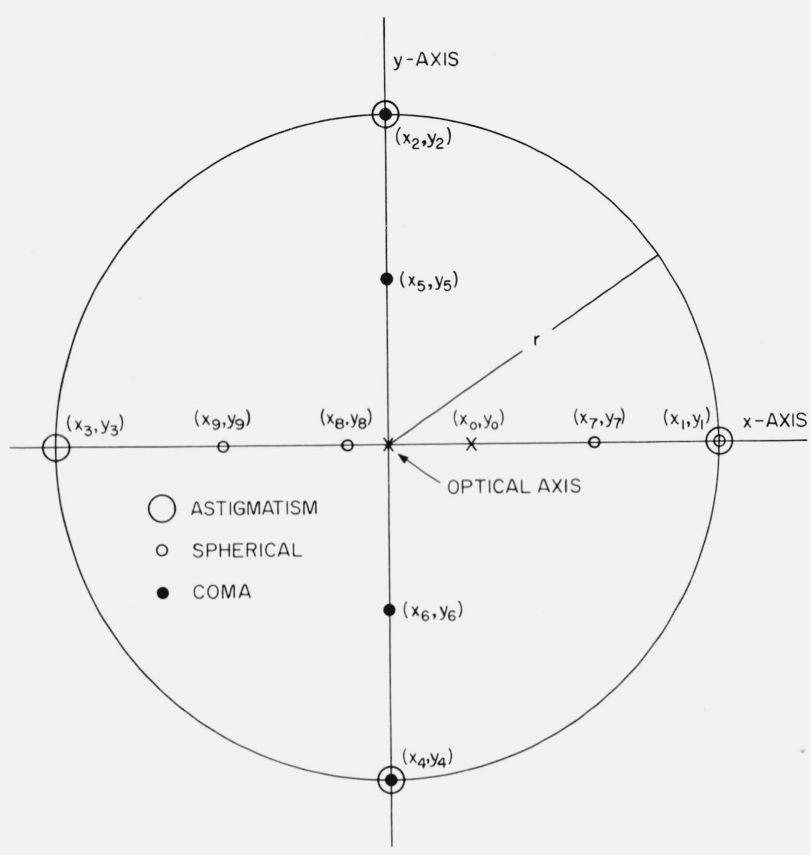

Figure 3. The distribution of reference points for most precise evaluation of primary aberrations.

The four larger circles, on the axes, are for astigmatism; the four smaller circles, on the $x$-axis, are for spherical aberration; and the four solid disks, on the $y$-axis, are for coma.

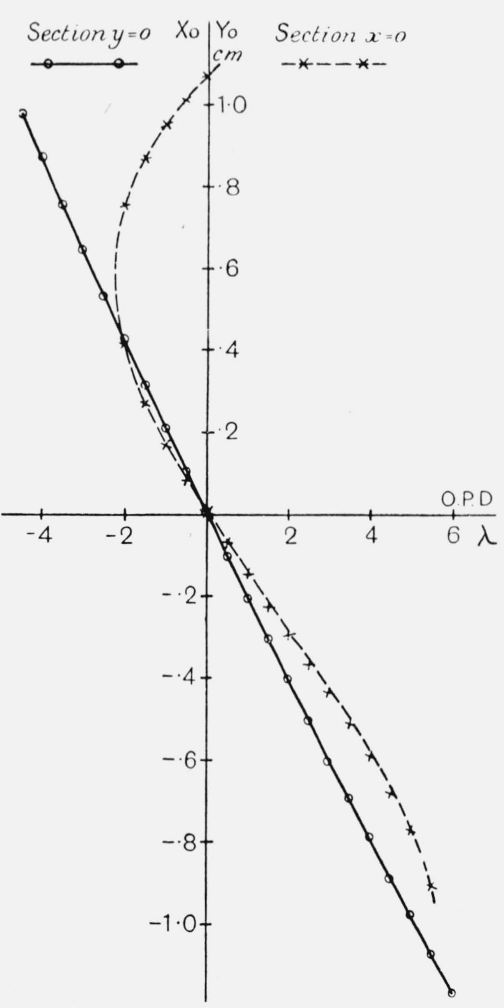

FIGURE 4. Cross sections of the wave front along the $\mathrm{x}$ and $\mathrm{y}$-axes. 
TABLE 1. Value for astigmatism, C, and probable error, $\Delta \mathrm{C}$, (in wavelength) for different lens apertures (in millimeters)

\begin{tabular}{c|c|c|c|c|c|c}
\hline \hline $\begin{array}{c}\text { Radius } \\
\text { vector }\end{array}$ & \multicolumn{3}{c|}{ Orders of interference } & \multicolumn{2}{c}{$\begin{array}{c}\text { Coefficient of } \\
\text { astigmatism }\end{array}$} \\
\hline & & $p_{2}$ & $p_{3}$ & $p_{4}$ & $C$ & $\Delta C$ \\
$r$ & & & & & & \\
\hline & & & & & & \\
1 & -0.48 & -0.63 & 0.48 & 0.68 & 1.250 & 0.650 \\
2 & -.96 & -1.17 & .96 & 1.32 & 0.938 & .163 \\
3 & -1.43 & -1.61 & 1.46 & 2.04 & 1.111 & .072 \\
4 & -1.89 & -1.97 & 1.97 & 2.74 & 1.078 & .041 \\
5 & -2.35 & -2.20 & 2.47 & 3.41 & 1.090 & .026 \\
6 & -2.80 & -2.28 & 2.98 & 4.04 & 1.097 & .018 \\
7 & -3.25 & -2.15 & 3.52 & 4.60 & 1.112 & .013 \\
8 & -3.70 & -1.82 & 4.06 & 5.08 & 1.133 & .010 \\
9 & -4.15 & -1.32 & 4.58 & 5.45 & 1.142 & .008 \\
10 & -4.61 & -0.53 & 5.13 & 5.74 & 1.173 & .007 \\
11 & -5.13 & +.48 & 5.66 & 5.86 & 1.200 & .005 \\
\hline
\end{tabular}

Since the fringes in figure 2 are precisely represented by eq (1), the values of the corresponding parameters should be independent of the choice of reference points. The value for $C$ (labeled $C_{k}$ in fig. 5) obtained from any family of four reference points in figure 2 will be the same as that found by Kingslake because this value was used for computing these curves. The corresponding probable error in $C_{k}$ (i.e., $\Delta C_{k}$ ) given by Kingslake is also indicated in figure 5 to illustrate the relative probable error for results obtained from four favorably chosen reference points and from 44 reference points treated statistically by the Method of Least Squares. Since the coefficient, $C$, varies with $r$ (i.e., from one family of reference points to another) eq (1) obviously does not apply to the fringe pattern of figure 1 . The lens that produced this set of fringes is obviously afflicted with higher order aberrations.

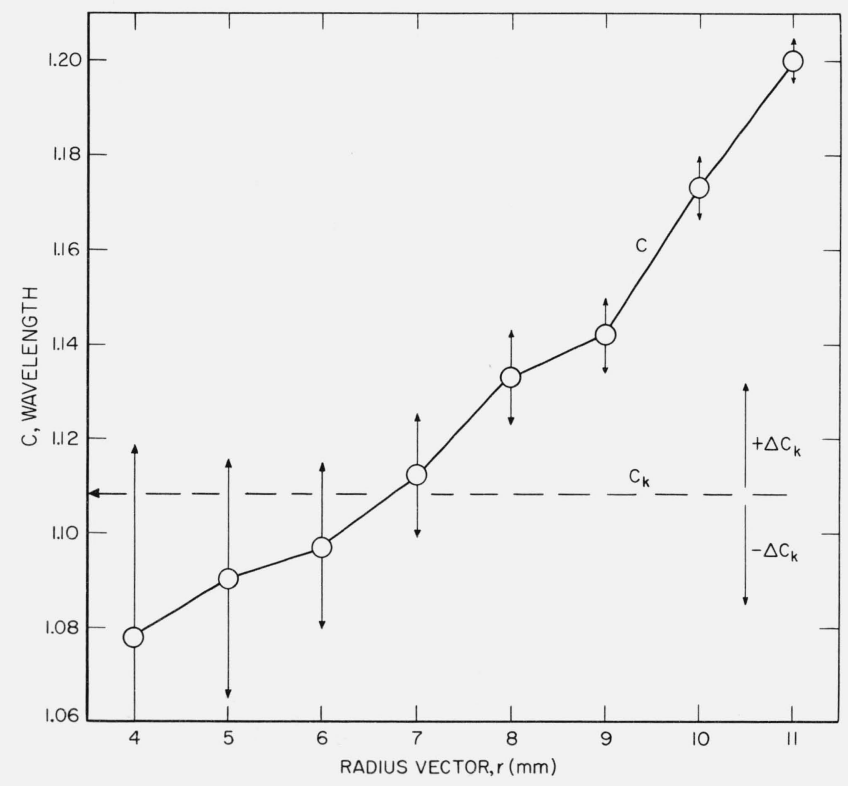

FigURE 5. Values of the coefficient of astigmatism, C, versus semiaperture, $\mathrm{r}$, of the lens.

The vertical arrows represent computed probable errors in $C$. The horizontal dashed line represents the value for $C$ obtained from figure 2 and given in footnote 2.

\section{Coma}

Coma cannot be evaluated from a family of reference points that are all equally distant from the origin, as is the case for astigmatism. However, if we choose a family of four points on the $y$-axis (fig. 3) made up of two pairs such that the two points of each pair are equally distant from the origin but of opposite sign, then we can obtain an equation for $B$ as a function of the coordinates of these four points. If the ordinates of the inner pair of points are one half the value of those for the outer points and the outer points are near the margin of the lens the resultant value for $B$ will be the most precise value obtainable from any family of four points. The marginal point should be far enough from the margin to permit an accurate evaluation of the corresponding order of interference.

The observed $x, y, p$-coordinates for the four points are $\left(x_{2}, y_{2}, p_{2}\right),\left(x_{5}, y_{5}, p_{5}\right),\left(x_{6}, y_{6}, p_{6}\right)$, and $\left(x_{4}, y_{4}, p_{4}\right)$, where

$$
\left.\begin{array}{l}
x_{2}=x_{5}=x_{6}=x_{4}=0 \\
y_{2}=2 y_{5}=-2 y_{6}=-y_{4}=r
\end{array}\right\} .
$$

We may substitute the coordinates for each of these four points into eq (1) and obtain four equations, from which we may obtain the following formula for $B$ :

$$
3 r^{3} B=2\left(p_{2}-p_{4}+2 p_{6}-2 p_{5}\right) .
$$

If we compute values for $B$ that correspond to different values of $r$ (i.e., for different apertures of the lens) we obtain the results shown in figure 6 . The

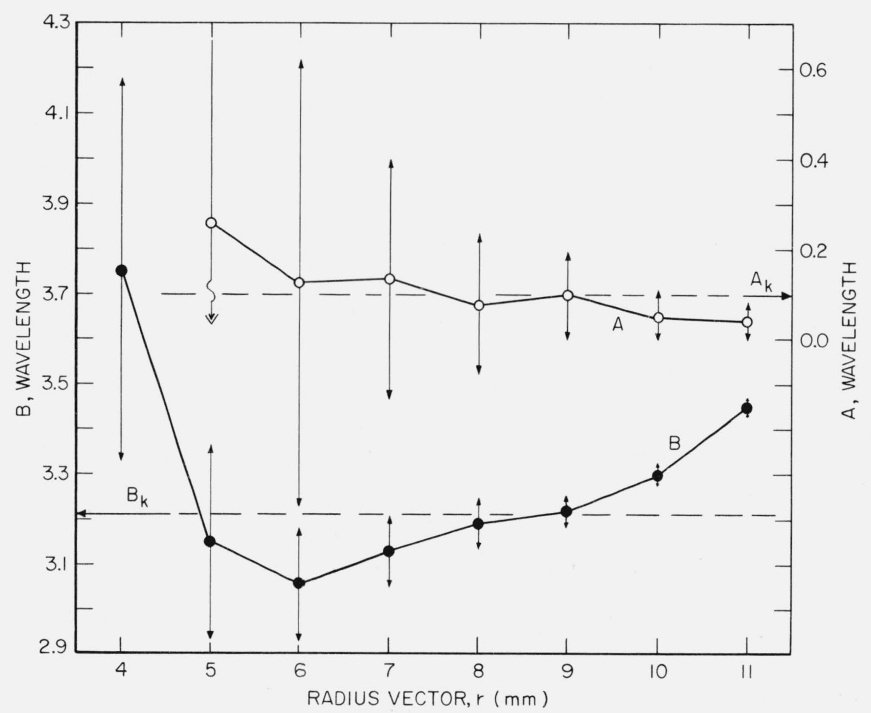

Figure 6. Values of the coefficients of coma, B (on the left) and spherical aberration, A (on the right) versus the semiaperture, $\mathrm{r}$, of the lens.

The vertical arrows represent computed probable errors. The horizontal, dashed lines represent values obtained from figure 2 and given in footnote 2 . 
probable error, $\Delta B$, of $B$ (based on the same assumptions used for obtaining $\Delta C$, above) is indicated by the vertical arrows attached to the corresponding points.

The value of $B$, computed from figure 2, (indicated as $B_{K}$ in fig. 5), is constant for all families of reference points. This value for $B_{K}$ is the same as that given by Kingslake for the same lens.

\section{Spherical Aberration}

Spherical aberration cannot be evaluated from a family of reference points that are equally distant from the origin, as is the case for astigmatism; nor can it be evaluated from a family of four points on the $y$-axis, as is the case for coma. However, if we choose a family of four points on the $x$-axis, made up of two pairs such that the two points of each pair are equally distant from some convenient point, $x_{0}$ (see fig. 3) but in opposite directions from $x_{0}$, then we can obtain an equation for $A$ as a function of the coordinates of these four points alone. Also, if the distance from $x_{0}$ to each of the inner pair of points $\left(x_{7}\right.$ and $\left.x_{8}\right)$ are one-half the value of those for the outer points $\left(x_{1}\right.$ and $x_{9}$ ), and the outermost point, $x_{1}$, is near the margin of the lens, the resultant value for $A$ will be the most precise value obtainable from any such family of points.

The $x, y, p$-coordinates of the four points are $\left(x_{1}\right.$, $\left.y_{1}, p_{1}\right),\left(x_{7}, y_{7}, p_{7}\right),\left(x_{8}, y_{8}, p_{8}\right)$, and $\left(x_{9}, y_{9}, p_{9}\right)$, where

$$
\left.\begin{array}{l}
x_{1}=(8 / 5) x_{7}=4 x_{0}=-8 x_{8}=-2 x_{9}=r, \\
y_{1}=y_{7}=y_{0}=y_{8}=y_{9}=0
\end{array}\right\} .
$$

This family of points has the same relationship to $x_{0}$ as the family of points in eq (5) has to the origin (optic axis).

On successively substituting the coordinates of these four points into eq (1) we obtain four equations from which we may obtain the following formula for $A$ :

$$
81 A r^{4}=128\left[p_{1}-p_{9}+2\left(p_{8}-p_{7}\right)\right] .
$$

Values for $A$, computed for different values of $r$ (i.e., for different apertures) are shown in figure 6, together with the corresponding probable errors, $\Delta A$. The value for $A$, computed from figure 2 (indicated as $A_{K}$ in fig. 6), is constant for all values of $r$. The value $A_{K}$ is that given by Kingslake for the same lens.

\section{Conclusions}

The method for obtaining the coefficients of primary, monochromatic aberrations, described here, requires a very small amount of calculations. A 10-in. slide rule is adequate for most work.

The coefficients of all three of the aberrations are found to vary with the aperture of the lens. This indicated that the method is sufficiently precise to evaluate even higher orders of aberrations provided the necessary formula for optical path difference is available. 